\title{
Coronal Activity on Rapidly-Rotating Solar-Like Stars: Chandra Observations of ER Vul
}

\author{
Alexander Brown ${ }^{1}$, Joanna M. Brown ${ }^{2}$, Rachel A. Osten ${ }^{1,3}$, Thomas R. \\ Ayres $^{1}$, and Edward Guinan ${ }^{4}$ \\ ${ }^{1}$ Center for Astrophysics and Space Astronomy, University of Colorado, \\ Boulder, CO 80309-0389, USA \\ ${ }^{2}$ Department of Astronomy, California Institute of Technology, \\ Pasadena, CA 91125, USA \\ ${ }^{3}$ National Radio Astronomy Observatory, Charlottesville, VA \\ 22903-2475, USA \\ ${ }^{4}$ Department of Astronomy, Villanova University, Villanova, PA 19085, \\ $U S A$
}

\begin{abstract}
We investigate the coronal structure of rapidly-rotating, solar-like stars using Chandra HETGS spectra of the short-period binary ER Vul, and by comparison with X-ray observations of the Sun and other dwarf stars. ER Vul consists of two solar-like (G0 + G5) dwarfs with rotation rates $\sim 40$ times that of the Sun. This binary is not interacting and these stars are the fastest rotating $\mathrm{G}$ dwarfs suitable for high resolution X-ray spectroscopy. X-ray (1.8$40 \AA$ ) spectra were obtained on 2001 March 29-30 along with 10.5 hours of simultaneous VLA monitoring at 3.6 and $20 \mathrm{~cm}$. These spectra show hot, multitemperature coronal emission with emission lines ranging in temperature from O VII (2 MK) to Fe XXIV (30 MK). ER Vul showed only low-level variability during the X-ray observation. Unlike the behaviour of longer period active binaries, no large, long-duration flares were detected, consistent with previous $\mathrm{X}$-ray observations of this binary. No evidence for eclipses is seen in either the Xray or radio emission. The coronal emission measure distribution and elemental abundances were derived for ER Vul.
\end{abstract}

\section{Introduction}

Simultaneous multi-wavelength observations of stellar flares and other coronal variability offer an extremely powerful technique to determine the physical processes controlling thermal and nonthermal coronal plasmas. Flares occur frequently on active binary stars (Osten \& Brown 2000), with most systems spending $30-40 \%$ of the time flaring. ER Vul consists of two solar-like stars in a short-period $\left(\mathrm{P}_{\text {orb }}=0.694 \mathrm{~d}\right)$ detached binary system. Due to tidal effects, both stars are rapid rotators with rotational velocities of 81 and $71 \mathrm{~km} \mathrm{~s}^{-1}$ (Hill et al. 1990). Thus, these stars are just like the Sun but are rotating at $\sim 40$ times the solar rate and consequently show extremely strong stellar activity. 
Table 1. Coronal Elemental Abundances Relative to Iron and Hydrogen

\begin{tabular}{ccccccc}
\hline & \multicolumn{2}{c}{ ER Vul } & \multicolumn{2}{c}{ AU Mic } & \multicolumn{2}{c}{ AD LeO } \\
\hline Element & $\mathrm{A} / \mathrm{Fe}$ & $\mathrm{A} / \mathrm{H}$ & $\mathrm{A} / \mathrm{Fe}$ & $\mathrm{A} / \mathrm{H}$ & $\mathrm{A} / \mathrm{Fe}$ & $\mathrm{A} / \mathrm{H}$ \\
\hline $\mathrm{Fe}$ & $\ldots$ & $0.55 \pm 0.05$ & $\ldots$ & $0.42 \pm 0.10$ & $\ldots$ & $0.56 \pm 0.12$ \\
$\mathrm{~N}$ & $\ldots$ & $\ldots$ & $1.75 \pm 0.68$ & $0.74 \pm 0.34$ & $0.45 \pm 0.32$ & $0.25 \pm 0.19$ \\
$\mathrm{O}$ & $0.53 \pm 0.05$ & $0.29 \pm 0.04$ & $1.35 \pm 0.08$ & $0.57 \pm 0.14$ & $0.55 \pm 0.04$ & $0.31 \pm 0.07$ \\
$\mathrm{Ne}$ & $1.26 \pm 0.10$ & $0.69 \pm 0.08$ & $3.55 \pm 0.40$ & $1.49 \pm 0.39$ & $2.39 \pm 0.34$ & $1.34 \pm 0.35$ \\
$\mathrm{Mg}$ & $1.05 \pm 0.07$ & $0.58 \pm 0.07$ & $0.54 \pm 0.07$ & $0.23 \pm 0.06$ & $0.71 \pm 0.12$ & $0.40 \pm 0.11$ \\
$\mathrm{Al}$ & $2.6 \pm 0.5$ & $1.4 \pm 0.3$ & $4.6 \pm 1.5$ & $1.93 \pm 0.78$ & $3.8 \pm 2.3$ & $2.13 \pm 1.37$ \\
$\mathrm{Si}$ & $0.81 \pm 0.06$ & $0.45 \pm 0.03$ & $0.94 \pm 0.10$ & $0.40 \pm 0.10$ & $1.34 \pm 0.18$ & $0.75 \pm 0.19$ \\
$\mathrm{~S}$ & $0.66 \pm 0.10$ & $0.36 \pm 0.07$ & $1.3 \pm 0.6$ & $0.55 \pm 0.29$ & $\ldots$ & $\ldots$ \\
\hline
\end{tabular}

\section{Observations}

We observed ER Vul with Chandra for $112 \mathrm{ksec}$ on 2001 March 29-30, using the High Energy Transmission Grating Spectrometer (HETGS) with the ACIS-S detector. The HETGS disperses photons through two concentric sets of transmission gratings: the Medium Energy Grating (MEG) covers the wavelength range 1.7-40 $\AA$ in first order, while the High Energy Grating (HEG) covers the wavelength range 1.7-20 $\AA$ in first order, with almost twice the spectral resolution of the MEG in the regions of overlap. The X-ray pointing was coordinated with a 10.5 hour $(\sim 0.4 \mathrm{~d})$ VLA observation at 3.6 and $20 \mathrm{~cm}$ to study the effects of flaring on both the thermal and nonthermal coronal electron populations.

\section{Results}

The Chandra spectra show a multi-temperature spectrum with emission lines ranging in temperature from O VII (2 MK) to Fe XXIV (30 MK). Emission lines seen include the hydrogen- and helium-like lines of $\mathrm{O}, \mathrm{Ne}, \mathrm{Mg}, \mathrm{Al}, \mathrm{Si}$, and $\mathrm{S}$. Numerous iron lines are present spanning the ionization stages Fe XVII to Fe XXIV. The lightcurves of the MEG and HEG photons show only low-level variability and no strong flares - the "flare" reported by Brown et al. (2002) was, in fact, a background subtraction artifact. The coronal emission measure peaks near $10^{7} \mathrm{~K}$. The elemental abundances were measured relative to $\mathrm{H}$ (see Table 1) and compared to preliminary values for two active $M$ dwarfs. The helium-like triplets are too weak to allow determination of coronal electron densities.

Acknowledgments. This work is supported by Chandra grant GO1-2016X and NSF grant AST-0206367.

\section{References}

Brown et al. 2002, in Stellar Coronae in the Chandra and XMM-Newton Era, (eds. F. Favata \& J. Drake), ASP Conf. Ser. 277, p. 223

Hill, G., et al. 1990, A\&A 238, 145

Osten, R.A. \& Brown, A. 2000, ApJ 515, 746 